\title{
THE ASSESSMENT OF PROPERTIES OF THE INFORMATION FLOW IN THE SUBSYSTEM «THE MEASURING SENSOR - THE PROCESSING ELEMENT»
}

\author{
Victor Nikolaev ${ }^{1 \mathrm{a}}$, Anton Stuchkov ${ }^{2}$ \\ ${ }^{1}$ Southwest State University, 405000, Kursk, Russia \\ ${ }^{2}$ Tomsk State University, 634050, Tomsk, Russia
}

\begin{abstract}
Analyzed the scope and types of automated information systems containing subsystems of the "measuring sensor - element processing". On the basis of the Queuing theory a model of evaluating the properties of information flow in the subsystem. The time spent by the sensor data generation and processing time of the output data array to the processing elements described by an arbitrary distribution law. Using little's formula the analytical solution allows to estimate the required level of performance of the subsystem. The research of the obtained solution in the range of parameters of interest. On the basis of General theoretical solutions identified four private practical case, different values of the coefficients of variation of information flows.
\end{abstract}

\section{Introduction}

The present stage of the development of the society is characterized by the increasing role of innovative technologies, such as the measuring and information technologies, representing the set of the elements of infrastructure, the subjects of information system which are carrying out measurement, collecting, formation, distribution and use of diverse information in various fields of activity [1-5].

The basic subsystems of the type "the measuring sensor - the processing element" are the most widespread in the automated information systems. Such subsystems are always a component of the automated systems in various fields of activity- science, education, industry, economy, medicine, social sphere [6-10].

In these conditions and the assessment of the properties of information flows in the automated system for the purpose of formation of requirements to parameters of standard elements is important and actual.

\footnotetext{
${ }^{\mathrm{a}}$ Corresponding author: nikovic54@yandex.ru
} 


\section{The theoretical part}

The analysis of literature shows that in the all-system plan, the basic subsystems of the type "the measuring sensor - the processing element" consist of the following basic elements $[11,12]$ :

1. The source (sensor) - the initial elements of receiving of data or information on a state or properties of process, technology or system.

2. The receiver (processing element) - the initial elements which are a part of instruments of measuring and monitoring of information and property space.

Between these elements there is an information flow, the assessment or measurement of properties of which allows to form the requirements to these elements, in particular to the performance level (the capacity of the system) $[13,14]$.

Generally the initial input data flow from the sensor or measuring system comes to the element or the subsystem of processing. The time spent by the sensor on formation of data is described by unrestricted distribution law $A_{j}^{(i)}(t)$ and has the intensity

$$
\Lambda_{j}^{(i)}=d A_{j}^{(i)}(t) /\left(1-A_{j}^{(i)}(t)\right)
$$

The time of processing of output data array on the element of processing is distributed under the law $B_{j}^{(i)}(t)$ with the intensity

$$
M_{j}^{(i)}=d B_{j}^{(i)}(t) /\left(1-B_{j}^{(i)}(t)\right)
$$

Let us also suppose that the variation coefficients of the input flow $C_{A}$ and the time of data array staying at the processing stage $C_{M}$ is less than unity. As the results of researches on the analysis of temporary characteristics of processing in various automated systems have shown [11-14], such supposition is quite justified.

In the proposed formulation it is necessary, having the set restriction for time of formation of data array $T_{3}$, to determine the necessary level of capacity of the subsystem of processing (element) of the information system. Within the stated above and the accepted suppositions the task can be converted to the analysis of the queueing model. In this case the subsystem of processing is considered as single-channel queueing system with unbounded queue to which the unrestricted recurrent flow of applications comes in with the intensity $\Lambda$ and variation coefficient $0 \leq C_{\Lambda} \leq 1$. The intensity of processing also has the unrestricted distribution with average value $M$ and the variation coefficient $0 \leq C_{M} \leq 1$. For this case it is not posible to obtain the exact analytical decision, however it is possible to estimate the average queue length, to bound it above and below [14]

$$
\begin{aligned}
& \frac{\rho^{2}\left(C_{\Lambda}^{2}+C_{M}^{2}\right)}{2(1-\rho)}-\frac{\rho\left(1-C_{\Lambda}^{2}\right)}{2}+\rho \leq N_{c} \leq \\
& \leq \frac{\rho^{2}\left(C_{\Lambda}^{2}+C_{M}^{2}\right)}{2(1-\rho)}+\frac{(1-\rho)\left(1-C_{\Lambda}^{2}\right)}{2}+\rho
\end{aligned}
$$

where $N_{\mathrm{c}}$ is the average of the applications which are in the system; $\rho=\Lambda / M_{M}<1$ is the coefficient of the system utilization; $C_{A}$ is the variation coefficient of the input flow of applications; $C_{M}$ is the processing time variation coefficient.

As at the stage of the macroanalysis we are interested only in the top level of the capacity of the information subsystem providing the set data processing time $T_{3}$, then taking 
the right part (3) and using the Little's equation $N=\Lambda T$, we obtain the top value of time of formation of data array in the system

$$
T=\frac{\Lambda\left(C_{\Lambda}^{2}+C_{M}^{2}\right)}{M^{2} 2\left(1-\frac{\Lambda}{M}\right)}+\frac{\left(1-\frac{\Lambda}{M}\right)\left(1-C_{\Lambda}^{2}\right)}{2 \Lambda}+\frac{1}{M} .
$$

having made simple transformations with (4), we obtain the quadratic equation in $M$

$$
M^{2}\left(2 T \Lambda-1+C_{\Lambda}^{2}\right)-M 2 \Lambda\left(T \Lambda+C_{\Lambda}^{2}\right)+\Lambda^{2}\left(1-C_{M}^{2}\right)=0
$$

the solution of the equation (5) will be

$$
M_{1,2}=\frac{\Lambda\left(T \Lambda+C_{\Lambda}^{2}\right) \pm \Lambda \sqrt{\left(T \Lambda+C_{\Lambda}^{2}\right)^{2}-\left(2 T \Lambda-1+C_{\Lambda}^{2}\right)\left(1-C_{M}^{2}\right)}}{2 T \Lambda-1+C_{\Lambda}^{2}}
$$

Using the restriction for processing time $T \leq T_{3}$ and taking into account that we need the largest value of $M$, we obtain

$$
M \geq \Lambda \frac{\left(T_{3} \Lambda+C_{\Lambda}^{2}\right)+\sqrt{\left(T_{3} \Lambda+C_{\Lambda}^{2}\right)^{2}-\left(2 T \Lambda-1+C_{\Lambda}^{2}\right)\left(1-C_{M}^{2}\right)}}{2 T_{3} \Lambda-1+C_{\Lambda}^{2}}
$$

at $0 \leq C_{\Lambda} \leq 1$

$0 \leq C_{M} \leq 1$.

The expression (7) allows to determine the necessary capacity of the information subsystem as the stochastic macrosystem at the set parameters $\Lambda, T_{3}, C_{\Lambda}, C_{M}$.

\section{The practical part}

It is necessary to notice that the majority of the known laws by which the real distributions of an input flow and time of processing are approximated have variation coefficients less than unity. Therefore it is reasonable to select from (7) the special cases which are mostly often found in practice

$$
\begin{array}{lll}
1 & C_{\Lambda}=0 ; & M \geq \Lambda . \\
& C_{M}=0 ; & \\
2 & C_{\Lambda}=1 ; & M \geq \Lambda \frac{T_{3} \Lambda+1+\sqrt{\left(T_{3} \Lambda\right)^{2}+1}}{2 T_{3} \Lambda} . \\
& C_{M}=0 ; & \\
3 & C_{\Lambda}=0 ; & M \geq \frac{2 T_{3} \Lambda}{2 T_{3} \Lambda-1} . \\
& C_{M}=1 ; & \\
4 & C_{\Lambda}=1 ; & M \geq \Lambda+\frac{1}{T_{3}} \\
& C_{M}=1 ; &
\end{array}
$$

As it is seen from (8), in case of the determined input flow and constant time of processing the necessary capacity of the information subsystem depends only on the intensity of an input flow and should exceed it. The general time of staying in the system is defined only by the intensity of service as in this case the queues are not formed at the system input.

In other cases the type of dependence is more complex and significantly depends on time $T_{3}$, designated for the processing. So at values $\Lambda \approx 1 / T_{3}$ the level of intensity of processing of applications should only insignificantly exceed the value of intensity of the 
input flow. In case when $\Lambda_{\Lambda<</ T_{3}}$, the necessary system performance level should vastly exceed the level of intensity of the input flow. As it is shown by the analysis of expressions (8-11), the maximum value of level of capacity is obtained at the variation coefficients $C_{A}=1, C_{M}=1$ and exceeds the value of intensity of the input flow on the value inverse to the set processing time $T_{3}$. Therefore further it is reasonable to assess the top level of capacity of system at the information processing phase by the formula (11).

\section{Conclusion}

A mathematical model of mass service allowing to estimate the average queue length and load factor of the automated subsystem of the "sensor element" for various laws of distribution of internal information flows. The results of the study of this model allowed to identify the four most commonly used in practice, allows to substantiate the requirements of techno-economic characteristics of elements of the automated information-measuring systems at key phases of their life cycle.

\section{Acknowledgments}

The paper was written as part of the research project No. 8.2.31.2015, carried out with the support of the Program "Research Foundation of Tomsk State University named after D.I. Mendeleev" in 2015 - 2016, grant RFBR No. 16-29-04388/16.

\section{References}

[1] S. Staab, H. Stuckenschmidt, Semantic Web and Peer-to-Peer, Decentralized Management and Exchange of Knowledge and Information (Springer Science \& Business Media, New York, 2006)

[2] A. J. Bostel, V.K. Sagar, Computing and Control Engineering Journal 7, 169(1996)

[3] P. Viswanath, V. Anantharam, D. Tse, IEEE Transactions on Information Theory 4, 1968 (1999)

[4] Y. Zhu, Multivariable System Identification for Process Control (Elsevier, The Netherlands, 2011)

[5] J.A. Hanley, A. Negassa, M.D.D.B. Edwardes, J.E. Forrester, American Journal of Epidemiology 157, 364 (2003) doi: 10.1093/aje/kwf215

[6] Y. Yagi, J.R. Gilbertson, Journal of Telemedicine and Telecare 11, 109(2005) doi:10.1258/1357633053688705

[7] A.A. Maslak, V.N. Nikolaev, Information and measuring and operating systems 11, 46 (2013)

[8] O.G. Diev, K.S. Dyagterev, V.N. Nikolaev, High technologies 15, 21 (2014)

[9] V.N. Nikolaev, A.A.B. Dafalla, L.V. Pleskanos, K.D.A. Kassim, Telecommunications and Radio Engineering 72, $1219 \quad$ (2013) doi: 10.1615/TelecomRadEng.v72.i13.80

[10] A.R. Ward, P.W. Glinnj, Queueing Systems 50, 371 (2005) doi: 10.1007/s11134005-3282-3

[11] R. Yuan, W. T. Strayer, Virtual Private Networks: Technologies and Solutions (Addison-Wesley, Strayer, 2001)

[12] P. G. Harrison, Queueing Systems 41, 271 (2002) doi: 10.1023/A:1015842221910 
[13] T. L. Saaty, Elements of Queuing theory with applications (McGraw-Hill, New York, 1961)

[14] D.G. Kendall, Ann. Math. Statist. 24, 338 (1953) 